\title{
Schätzung der Ausgaben der öffentlichen Hand durch den Konsum illegaler Drogen in Deutschland
}

\author{
Public Expenditure Caused by the Consumption of Illicit Drugs in Germany
}

Autoren

Institute

\author{
S. Mostardt ${ }^{1}$, S. Flöter ${ }^{2}$, A. Neumann ${ }^{1}$, J. Wasem ${ }^{1}$, T. Pfeiffer-Gerschel ${ }^{2}$
}

${ }^{1}$ Universität Duisburg-Essen, Lehrstuhl für Medizin-Management

${ }^{2}$ IFT Institut für Therapieforschung, München

\author{
Schlüsselwörter \\ - illegale Drogen \\ - öffentliche Ausgaben \\ - Deutschland \\ Key words \\ - illicit drugs \\ - public expenditures \\ - Germany
}

\section{Bibliografie}

DOI http://dx.doi.org/

10.1055/s-0029-1243212

Online-Publikation: 11.1.2010

Gesundheitswesen 2010;

72: 886-894

(c) Georg Thieme Verlag KG

Stuttgart · New York

ISSN 0941-3790

\section{Korrespondenzadresse}

\section{S. Mostardt}

Alfried Krupp von Bohlen und Halbach-Stiftungslehrstuhl für Medizin-Management Universität Duisburg-Essen Standort Essen Fachbereich Wirtschaftswissenschaften

Schützenbahn 70

45127 Essen

sarah.mostardt@

medman.uni-due.de

\section{Zusammenfassung \\ V}

Ziel: Für Deutschland fehlt eine wissenschaftlich fundierte und umfassende Übersicht darüber, wie hoch die von der öffentlichen Hand für den Gesamtbereich ,illegale Drogen“ aufgewendeten Ausgaben sind. Ziel des vorgestellten Projektes ist es, erstmals in Deutschland eine umfassende Schätzung der direkten (gekennzeichneten und nicht-gekennzeichneten) Ausgaben der öffentlichen Haushalte in Bezug auf den Missbrauch und die Abhängigkeit von illegalen Drogen für das Jahr 2006 vorzunehmen.

Methodik: Je nach Ausgabenträger erfolgte ein unterschiedliches Vorgehen bei der Datensammlung. Die Haushaltspläne und Statistiken der Gebietskörperschaften wurden nach relevanten Daten durchsucht sowie Behörden schriftlich befragt. Die Erhebung der Ausgaben der Sozialversicherungsträger erfolgte über eine schriftliche Befragung. Neben der Rentenversicherung Bund wurden die 40 größten gesetzlichen Krankenkassen mittels standardisierter Fragebögen angeschrieben, deren Angaben für die gesamte gesetzliche Krankenversicherung (GKV) extrapoliert wurden.

Ergebnisse: Auf Ebene der Gebietskörperschaften konnte ein Betrag zwischen 3,7 und 4,6 Mrd. € ermittelt werden, der für die Bekämpfung des Drogenproblems (in Form von Präventions-, Interventions- und Repressionsmaßnahmen) ausgegeben wird. Die Gesamtausgaben der Rentenversicherung im Zusammenhang mit illegalen Drogen in Form von medizinischen Rehabilitationen, Leistungen zur Teilhabe am Arbeitsleben und Renten wegen Erwerbsminderung betragen etwa 171,7 Mio.€. Die Hochrechnung der Ausgaben der gesetzlichen Krankenkassen für Medikation, Krankenhausaufenthalte, Rehabilitationen usw. im Zusammenhang mit illegalen Drogen ergab 1,4 Mrd.€.

\section{Abstract \\ $\checkmark$}

Aim: A scientifically based overview of public expenditures related to illicit drugs was lacking for Germany. The aim of the present project is to carry out for the first time a comprehensive estimation of direct (labelled and non-labelled) expenditures of the government and the social insurance funds related to the use of illicit drugs in Germany for the year 2006.

Methods: Depending on the respective financing bodies, different ways of data collection were required. Data on drug-related expenditure were searched in publically available budget documents and statistical reports; moreover written requests were sent to the relevant public authorities. Information on the expenditures of social insurance funds was collected through standardised questionnaires, which were sent to the Statutory Pension Insurance Scheme (Rentenversicherung Bund) and the 40 biggest statutory health insurance companies. The collected data were extrapolated for the total statutory health insurance.

Results: All in all, on the government side an amount of 3.7-4.6 billion Euro spent on the task of tackling illicit drugs (i.e., for prevention, intervention and law enforcement measures) was identified. The expenditures of the pension funds related to illegal drug use amount to 171.7 million Euro. For the statutory health insurances a total expenditure of 1.4 billion Euro was estimated.

Conclusion: The aim of the project - a first estimation of the public expenditures concerning illicit drugs in Germany - has been achieved. Nevertheless, there remains some degree of uncertainty regarding the overall result because of the heterogeneous data quality. The approximation of the amount of public expenditures concerning illegal drug use still gives no information about adequate spending or actual benefits. However, 
Schlussfolgerungen: Das Ziel des Projektes - eine erste Abschätzung der öffentlichen Ausgaben im Kontext von Missbrauch und Abhängigkeit von illegalen Drogen - konnte erreicht werden. Anzumerken ist, dass zweifelsohne Unsicherheiten bezüglich des Gesamtergebnisses aufgrund der heterogenen Datenqualität bestehen. Das Ergebnis gibt noch keinen Aufschluss über die Angemessenheit und den tatsächlichen Nutzen der aufgewendeten öffentlichen Mittel. Es bildet allerdings die unabdingbare Grundlage für eine solche Bewertung und leistet einen wichtigen Beitrag zur Versachlichung der Diskussion.

\section{Einleitung}

\section{Relevanz der Fragestellung}

Der Konsum von illegalen Drogen ist mit einer hohen Mortalität und Morbidität behaftet [1]. Über die Höhe der Ausgaben für die öffentliche Hand, die mit dem Missbrauch illegaler Drogen einhergehen ist bislang wenig bekannt. In diesem Zusammenhang formuliert der Drogenaktionsplan 2005-2008 der Europäischen Union [2] als ein Ziel die Schätzung der öffentlichen Ausgaben im Drogensektor. Ein erster Versuch der systematischen Methodenentwicklung findet sich im Selected Issue 2008 der Europäischen Beobachtungsstelle für Drogen und Drogensucht (EMCDDA) [3]. Zehn Länder der Europäischen Union haben diese Aufstellung der öffentlichen Ausgaben im Drogensektor bereits teilweise erarbeitet. Für Deutschland existierte eine solche Aufstellung bisher nicht. Es erfolgte lediglich eine Abschätzung einzelner Kostenbereiche für das Jahr 2001 im Rahmen des ReitoxBerichts [4]. Eine ältere Abschätzung der Kosten im Zusammenhang mit sogenannten „harten Drogen“, womit vor allem Opiate/ Heroin gemeint waren, erfolgte durch Hartwig \& Pies für das Jahr 1992 [5]. Schätzungen jüngeren Datums liegen nicht vor. Ein weiterer wesentlicher Faktor für die Relevanz der vorliegenden Arbeit ist der zunehmende Eingang gesundheitsökonomischer Perspektiven in das Gesundheitswesen. Grund hierfür ist die Begrenztheit der dem Gesundheitswesen zur Verfügung stehenden Mittel. Der demografische Wandel sowie der technologische Fortschritt werden als grundsätzliche Ursachen für den Kostenanstieg verantwortlich gemacht. Hinzu kommt die weitgehende Stagnation der Einnahmeseite [6]. Es stellt sich somit die Frage, wie die wenigen finanziellen Mittel effizient verteilt werden können. Hierbei ist eine Schätzung der Ausgaben der öffentlichen Hand aufgrund des Konsums illegaler Drogen in Deutschland von erheblichem Interesse, schafft sie doch die informationelle Grundlage für eine Diskussion über den effizienten Einsatz von Ressourcen im Bereich der Suchthilfe und -bekämpfung im Zusammenhang mit illegalen Drogen.

\section{Begriffsdefinitionen}

Ein zentraler Punkt bei der Durchführung dieser Schätzung ist die klare Abgrenzung der Kostenarten, die in der Analyse berücksichtigt werden. Um die Vergleichbarkeit der Ergebnisse innerhalb der Europäischen Union zu gewährleisten, orientiert sich die vorliegende Schätzung an den Vorschlägen der Europäischen Beobachtungsstelle für Drogen und Drogensucht (EBDD) [3].

Berücksichtigt werden bei der Schätzung ausschließlich direkte Kosten, also monetäre/finanzielle Kosten, die im direkten $\mathrm{Zu}-$ sammenhang mit illegalen Drogen stehen. Indirekte Kosten in Form von Produktivitätsverlusten aufgrund frühzeitiger Morta- it provides the indispensable basis for such an assessment and contributes to a more objective discussion. lität oder Folgeerkrankungen aufgrund des Drogenkonsums, werden nicht mit in die Analyse einbezogen.

Desweiteren unterscheidet man zwischen öffentlichen und privaten Ausgaben. Der Begriff „öffentliche Ausgaben“ bezieht sich auf den Wert der Güter und Dienstleistungen, die vom Staat im Rahmen der Wahrnehmung seiner einzelnen Aufgaben eingesetzt werden. Kosten der privaten Haushalte werden nicht berücksichtigt.

In die folgende Schätzung werden zudem „gekennzeichnete“ und „nicht-gekennzeichnete“ Ausgaben miteinbezogen. Gekennzeichnete Ausgaben sind Ausgaben für (illegale) Drogen, die im Haushaltsplan oder anderen öffentlich zugänglichen Dokumenten als solche gekennzeichnet sind (z.B. Drogenbeauftragte, Ausgaben für Suchtkrankenhilfe). Nicht-gekennzeichnete Ausgaben umfassen Ausgaben in Bezug zu illegalen Drogen, die nicht als solche gekennzeichnet sind, da sie in andere Programme/ Maßnahmen mit weiter gefassten Zielen eingebettet sind (z.B. Polizei, Strafvollzug, Entwicklungshilfe).

\section{Ziel}

Es soll erstmals eine umfassende Schätzung und Beschreibung der direkten (gekennzeichneten und nicht-gekennzeichneten) Ausgaben der öffentlichen Hand in Deutschland in Bezug auf den Missbrauch und die Abhängigkeit von illegalen Drogen für das Jahr 2006 vorgenommen werden.

\section{Methodik}

\section{$\nabla$}

Im Anschluss an die Festlegung der Ausgabenarten, die in die Analyse miteinbezogen werden, wurden diejenigen Behörden und Institutionen identifiziert, die diese Ausgaben tätigen. Das sind zum einen die Gebietskörperschaften (Bund, Länder und Kommunen) und zum anderen die Sozialversicherungsträger (Krankenversicherung, Rentenversicherung und Unfallversicherung). Bei der Datensammlung musste je nach Ausgabenträger unterschiedlich vorgegangen werden.

Im Bereich der Gebietskörperschaften wurden zunächst der Bundeshaushalt sowie die Haushaltspläne der Länder mittels einer Volltextsuche in den öffentlich im Internet zugänglichen Dokumenten nach relevanten Daten durchsucht ${ }^{1}$. Im Anschluss daran wurden das Bundesministerium für Gesundheit sowie die Drogenbeauftragten der Länder schriftlich befragt. Sie wurden gebeten die identifizierten Ausgaben zu bestätigen, gegebenenfalls Anteile für illegale Drogen zu spezifizieren und weitere Ansprechpartner zu nennen, die über weitere Informationen verfügen könnten. Mithilfe dieses Schneeballprinzips wurden wei-

\footnotetext{
${ }^{1}$ Verwendete Suchbegriffe: „Sucht*“, „Droge*“, „Rauschgift*“, „Betäubungsmittel*“, ,Substitution*“, ,Maßregel“*”
} 
tere relevante Ministerien, Referate und Institutionen identifiziert und ebenfalls schriftlich befragt. Da es aufgrund der großen Anzahl von Kommunen in Deutschland praktisch unmöglich war, ebenfalls sämtliche kommunalen Haushalte zu analysieren, wurden hier die drei kommunalen Spitzenverbände (Deutscher Landkreistag, Deutscher Städtetag, Deutscher Städte- und Gemeindebund) sowie 44 kommunale Landesverbände angeschrieben und um Informationen zu Ausgaben mit Bezug zu illegalen Drogen gebeten.

Im Laufe der Datensammlung wurde deutlich, dass vor allem im Bereich staatlicher Repressionen im Zusammenhang mit illegalen Drogen kaum spezifische Daten in den Haushaltsdokumenten zur Verfügung stehen. Deshalb wurde nach alternativen Schätzverfahren für solche Ausgaben gesucht, die aufgrund drogenbezogener Straftaten entstehen und vor allem die staatlichen Funktionen Polizei, Gerichte und Strafvollzug betreffen. Angelehnt an eine frühere Studie von Hartwig \& Pies [5] wurden für diese Funktionen Schätzungen anhand von Sekundärdaten des Statistischen Bundesamtes und des Bundeskriminalamtes vorgenommen.

Für die Funktionsbereiche „Polizei“, „Gerichte“ und „Strafvollzug“ lagen vom Statistischen Bundesamt jeweils Angaben zu den Gesamtausgaben des Staates vor (17,36Mrd.€ für Polizei; 10,09 Mrd.€ für Gerichte; 2,63 Mrd.€ für Strafvollzug; [7]). Anhand weiterer vorliegender Daten und Statistiken, die im Folgenden kurz aufgezählt werden, wurde dann der Anteil berechnet, der davon dem Bereich „illegale Drogen“ zugeordnet werden kann.

Um die drogenbezogenen Ausgaben für den Bereich „Polizei“ zu schätzen, lagen zum einen die Gesamtzahl der registrierten Straftaten oder alternativ die Gesamtzahl der aufgeklärten Straftaten vor, zum anderen der Anteil der Rauschgiftdelikte nach dem Betäubungsmittelgesetz (BtMG) und der Anteil der durch Drogenkonsumenten begangenen Straftaten an der Gesamtzahl der Straftaten [8]. Für den Bereich „Gerichte“ wurden Daten zur Anzahl insgesamt abgeurteilter Personen, sowie zum Anteil der Abgeurteilten nach BtMG [9] bzw. zum Anteil der durch Drogenkonsumenten begangenen Straftaten [8] herangezogen. Für den Bereich „Strafvollzug“ lagen alternativ Angaben zur Anzahl verurteilter Personen inklusive Länge der Haftstrafe (nur früheres Bundesgebiet inkl. Gesamt-Berlin, [9]), zur Anzahl der Personen im Strafvollzug zum Stichtag 31.3.2006 [10], und zu den Tageshaftkosten für einen Gefängnisplatz [11] vor.

Auch im Bereich kommunaler Ausgaben wurde nach alternativen Datenquellen gesucht. Für die ambulante Suchthilfe stehen Daten aus der Deutschen Suchthilfestatistik zur Verfügung [12]. In dieser Erhebung werden die teilnehmenden Einrichtungen unter anderem zu dem ihnen zur Verfügung stehenden Budget und dessen Herkunft befragt. Anhand zusätzlicher Daten zu den Hauptdiagnosen der behandelten Patienten und einer Extrapolation auf die geschätzte Gesamtzahl der ambulanten Suchthilfeeinrichtungen in Deutschland konnte somit ein Teil der kommunalen Ausgaben für illegale Drogen geschätzt werden.

Die Erhebung der Ausgaben der Sozialversicherungsträger erfolgte über eine schriftliche Befragung. Bei der Deutschen Rentenversicherung Bund wurde mittels eines standardisierten Fragebogens die Höhe der Ausgaben für medizinische Rehabilitationen, Leistungen zur Teilhabe am Arbeitsleben sowie Renten wegen Erwerbsminderung aller 16 deutschen Rentenversicherungsträger abgefragt. Die 40 größten gesetzlichen Krankenkassen wurden mittels standardisierter Fragebögen schriftlich nach ihren Ausgaben für Medikation, Krankenhausaufenthalte, Re- habilitationen, Kontakte zu Substitutionsärzten, Kontakte zu anderen Ärzten, Notarzteinsätze, Heilmittel, Hilfsmittel, Krankengeld, Aufenthalte in der Psychiatrie, Prävention und Selbsthilfe sowie Soziotherapie im Zusammenhang mit illegalen Drogen gefragt. Die Angaben der teilnehmenden Krankenkassen zu den Ausgaben wurden schließlich für die gesamte Gesetzliche Krankenversicherung anhand der Versichertenzahlen hochgerechnet.

Die gesetzliche Unfallversicherung als Bestandteil der Sozialversicherung versichert die Risiken aufgrund von Arbeitsunfällen einschließlich Wegeunfall sowie Berufskrankheiten. Der Konsum illegaler Drogen gilt für diese Risiken jedoch als Ausschlusskriterium für eine Kostenübernahme der Unfallversicherung, sodass hier keine relevanten Ausgaben für die Schätzung anfallen. Jedoch beinhalten die Ausgaben für Prävention der Unfallversicherung auch Projekte/Maßnahmen zur Suchtprävention. Es besteht bei der Deutschen Gesetzlichen Unfallversicherung (DGUV) aber keine Aufstellung der Präventionsprojekte zur Thematik der illegalen Drogen sowie deren Ausgaben. Aus diesen Gründen konnten die Ausgaben der Unfallversicherung in dieser Schätzung nicht berücksichtigt werden.

Da das Projekt auch in einen europäischen Rahmen eingebettet war, musste ein Weg gefunden werden, die Einzelergebnisse zusammenzuführen und mit den Ergebnissen anderer Länder vergleichbar zu machen. Auch für eine nationale Vergleichbarkeit von Zahlen im Zeitverlauf und eine mehr an Inhalten/Zielen statt an Kostenträgern ausgerichtete Zusammenstellung sollte ein System gefunden werden, um die einzelnen Teilergebnisse in konsistente Kategorien einzuordnen. Dafür wurde das international gebräuchliche Classification of the Functions of Government (COFOG) - System verwendet [13]. COFOG ist eine Klassifikation von Funktionen oder sozioökonomischen Zielen, die eine Regierung durch ihre Auslagen zu erreichen versucht. Sie wurde vom European System of National and Regional Accounts (ESA 95) implementiert und wird auch vom Statistischen Bundesamt zur Kategorisierung staatlicher Ausgaben verwendet. Die Klassifikation hat drei Gliederungsebenen: Auf der ersten Ebene ist sie untergliedert in zehn Funktionen (01. Allgemeine öffentliche Verwaltung, 02. Verteidigung, 03. Öffentliche Ordnung und Sicherheit, 04. Wirtschaftliche Angelegenheiten, 05. Umweltschutz, 06. Wohnungswesen und kommunale Einrichtungen, 07. Gesundheitswesen, 08. Freizeitgestaltung, Sport, Kultur und Religion, 09. Bildungswesen, 10. Soziale Sicherung). Diese werden auf der zweiten Gliederungsebene in 69 Gruppen aufgeteilt, die eine genauere Spezifikation der Ausgaben ermöglichen (z.B. 07.1 für Medizinische Erzeugnisse, Geräte und Ausrüstungen, 07.2 für ambulante Behandlung, 07.3 für stationäre Behandlung usw.). Eine weitere Untergliederung in Klassen ist möglich, wird aber bisher vom Statistischen Bundesamt nicht vorgenommen und auch in der vorliegenden Arbeit nicht berücksichtigt. Ein detailliertes Manual kann im Internet abgerufen werden [13]. COFOG erlaubt eine Betrachtung von zeitlichen Trends in den staatlichen Ausgaben für bestimmte Funktionen. Konventionelle Haushaltsdokumente sind dafür meist nicht geeignet, da sie eher die organisatorischen Strukturen der Regierung abbilden. Diese können sich jedoch mit der Zeit ändern. Außerdem ist eine Regierungsorganisation häufig für mehrere Funktionen zuständig und/oder die Verantwortlichkeit für eine Funktion liegt in den Händen mehrerer Organisationen. Das COFOG-System umgeht solche Schwierigkeiten und kann somit auch trotz organisatorischer Unterschiede eine Vergleichbarkeit zwischen verschiedenen Ländern herstellen. Dementsprechend wurden alle 
auf Ebene der Gebietskörperschaften und Sozialversicherungsträger erfassten Ausgaben den entsprechenden COFOG-Funktionen und -Gruppen zugeordnet.

\section{Ergebnisse}

Aufgrund der unterschiedlichen methodischen Vorgehensweisen, die vor allem in den unterschiedlichen Organisationsprinzipien begründet sind, werden im Folgenden die Ergebnisse der Gebietskörperschaften und der Sozialversicherungsträger getrennt dargestellt. Im Anschluss werden die Einzelergebnisse zusammengeführt und anhand der COFOG-Klassifikation nach inhaltlichen Gesichtspunkten zusammengefasst und dargestellt.

\section{Gebietskörperschaften}

Bund

Über die Volltextsuche im Bundeshaushalt 2006 konnten nur in den Budgets des Bundesministeriums für Gesundheit (BMG) und des Bundesministeriums für Finanzen (BMF) Ausgaben für illegale Drogen identifiziert werden. Auf Hinweis des BMG wurden zusätzlich die Bundesministerien des Innern (BMI), der Justiz (BMJ), für Arbeit und Soziales (BMAS), für Familie, Senioren, Frauen und Jugend (BMFSFJ), für Bildung und Forschung (BMBF) sowie für wirtschaftliche Zusammenarbeit und Entwicklung (BMZ) kontaktiert. Durch die schriftliche Anfrage konnten weitere relevante Ausgabentitel identifiziert werden, sodass sich auf Ebene des Bundes Gesamtausgaben in Höhe von 48,1 Mio.€ ergaben ( $\bullet$ Tab. 1). Das BMF wies in seinem Antwortschreiben darauf hin, dass der genannte Betrag keinen Anspruch auf Vollständigkeit erhebe. Das BMAS konnte keine Titel in direktem Zusammenhang mit illegalen Drogen benennen, da eine entsprechende Verwendung nicht aus der Zweckbestimmung hervorgehe. Das BMI konnte keine Informationen liefern.

\section{Länder}

\section{"Suchtbudgets" der Länder}

Bei der Analyse der Landeshaushaltspläne ergaben sich einige Schwierigkeiten vor allem dadurch, dass die Länder in ihrer Haushaltswirtschaft grundsätzlich selbständig und voneinander unabhängig sind. Dadurch werden Ausgabentitel mit Bezug zu Sucht und Drogen nicht einheitlich benannt, nicht in der gleichen Systematik erfasst und nicht in gleichem Maße detailliert beschrieben und aufgeschlüsselt. Es konnte dennoch in jedem Land eine Titelgruppe identifiziert werden, die als „Suchtbudget“ bezeichnet werden kann und die vor allem Ausgaben für Suchtkrankenhilfe, Suchtprävention, Forschungs- und Modellprojekte, sowie Koordinationsaufgaben im Bereich Sucht beinhaltete. Diese Titelgruppen waren in den meisten Fällen in den jeweiligen Gesundheits- und/oder Sozialministerien angesiedelt. Zu beachten ist, dass die Suchtbudgets sowohl Ausgaben für illegale als auch für legale Suchtmittel enthielten und die zusätzlichen Ausführungen nur selten eine eindeutige Zuordnung zuließen. Darüber hinaus wurde deutlich, dass die Länder das Subsidiaritätsprinzip unterschiedlich stark nutzen. Einige Haushalte enthielten auch Zuweisungen an Gemeinden und Gemeindeverbände, die die Gefahr der Doppelerfassung in sich bergen, wenn zusätzlich die Ausgaben der Kommunen erhoben werden.

Eine ausschließliche Zusammenstellung der eindeutig gekennzeichneten Ausgaben, d.h. der Ausgabentitel, aus deren Beschreibung klar hervorging, dass sie im Zusammenhang mit illegalen Drogen standen, resultierte in einem Betrag von 17,1 Mio.€ und beinhaltete vor allem die Ausgaben für das Substitutionsregister des Bundesinstituts für Arzneimittel und Medizinprodukte (BfArM), sowie einzelne Posten für Drogenkonsumräume, Hilfen für Substituierte u.ä. Da dieser Betrag die tatsächlichen Ausgaben deutlich unterschätzen würde, wurden die Drogenbeauftragten der Länder gebeten, einen prozentualen Anteil illegaler Drogen am jeweiligen Suchtbudget anzugeben. Eine solche Angabe lag zum Ende der Datenerhebung von zehn Bundesländern vor und zeigte eine sehr große Variationsbreite von 10,6$85 \%$. Für die sechs Länder, aus denen eine entsprechende Angabe fehlte, wurden drei verschiedene Schätzungen vorgenommen, um ein Intervall für die geschätzte Gesamtsumme der Ausgaben aller Länder angeben zu können: Eine Minimalschätzung nahm für die fehlenden Bundesländer einen Anteil illegaler Drogen am jeweiligen Suchtbudget von 10,6\% an, eine Schätzung anhand des Mittelwerts der zehn vorliegenden Angaben verwendete einen prozentualen Anteil von $43 \%$ und eine Maximalschätzung rechnete mit einem Anteil von 85\%. Daraus ergaben sich drei Schätzwerte für das Gesamt-Suchtbudget der Länder in Höhe von 45,9 Mio.€ (Minimalschätzung), 57,8 Mio.€ (Mittelwertschätzung) bzw. 73,1 Mio.€ (Maximalschätzung).

Ausgaben für den Maßregelvollzug

Ebenfalls in den Haushaltsplänen der Länder aufgeführt waren die Ausgaben für den Maßregelvollzug nach $\S \S 63,64$ Strafgesetzbuch (StGB). Dabei bezieht sich der $\S 64$ auf die Unterbringung suchtkranker Straftäter in einer Entziehungsanstalt. Die Landeshaushalte enthielten nur die Gesamtausgaben für den Maßregelvollzug, der Anteil der nach $\S 64$ untergebrachten Straftäter unter Ausschluss der Diagnose Alkoholabhängigkeit („Trunksucht“) ließ sich aber für zwölf Bundesländer aus Daten des Statistischen Bundesamtes berechnen [10] und reichte von 0 bis 31,5\%. Für ein weiteres Bundesland lag eine Angabe des Sozialministeriums zum entsprechenden Anteil drogenabhängiger Straftäter vor. Somit ergaben sich unter Ausschluss der drei Bundesländer, für die keine genaueren Daten vorlagen, Ausgaben für den Maßregelvollzug in Höhe von 159,9 Mio.€.

Weitere Ausgabentitel in den Länderhaushalten

Über die Volltextsuche ergaben sich vereinzelt weitere Ausgabentitel im Zusammenhang mit Sucht, z.B. einzelne Titel für Suchtprävention in Schulen. Dabei handelte es sich jedoch meistens um relativ geringe Beträge und der Anteil von illegalen Drogen an dem Betrag konnte nicht ermittelt werden. Da sich diese Titel zudem unsystematisch über die Länder verteilten, wurde entschieden, diese Einzelbeträge nicht in die Ausgabenaufstellung mit aufzunehmen.

Ausgaben für öffentliche Sicherheit und Ordnung (Polizei, Gerichte, Strafvollzug)

\section{Polizei}

Aus den vorliegenden Datenquellen ergaben sich zwei alternative Berechnungsansätze für die drogenbezogenen Ausgaben im Bereich „Polizei“: Wurde der Anteil registrierter Drogendelikte an der Gesamtzahl registrierter Straftaten als Kostenschlüssel verwendet und an die Gesamtausgaben angelegt, ergaben sich Ausgaben in Höhe von 1,68 Mrd.€. Der Anteil aufgeklärter Drogendelikte an der Gesamtzahl aufgeklärter Straftaten als Kostenschlüssel führte zu einer geschätzten Ausgabensumme von 2,17 Mrd.€. Dabei ist zu beachten, dass die vom Statistischen Bundesamt gelieferten Gesamtausgaben auch Ausgaben auf Bundes- und kommunaler Ebene enthalten (z. B. für die Bundespolizei oder die Sicherung der öffentlichen Ordnung in kommunaler Hand). Der Großteil der Polizeiausgaben liegt jedoch im Zuständigkeitsbereich der Länder. 
Tab. 1 Ausgaben des Bundes im Zusammenhang mit illegalen Drogen für das Jahr 2006.

\begin{tabular}{|c|c|c|c|c|}
\hline Ministerium & $\begin{array}{l}\text { Ausgaben in Bezug zu } \\
\text { illegalen Drogen in } €\end{array}$ & Zweckbestimmung & Quelle (n) & COFOG \\
\hline \multirow[t]{2}{*}{ Bundesministerium für Gesundheit (BMG) } & 25.000 & $\begin{array}{l}\text { Geschäftsstelle der Drogenbeauftragten } \\
\text { der Bundesregierung }\end{array}$ & $\begin{array}{l}\text { Bundeshaushalt + Angabe } \\
\text { des Ministeriums }\end{array}$ & 07.6 \\
\hline & 3.992 .000 & $\begin{array}{l}\text { Maßnahmen auf dem } \\
\text { Gebiet des Drogen- und } \\
\text { Suchtmittelmissbrauchs }\end{array}$ & $\begin{array}{l}\text { Bundeshaushalt } \\
+ \text { Angabe des } \\
\text { Ministeriums }\end{array}$ & $\begin{array}{l}07.4 \\
07.5 \\
07.6\end{array}$ \\
\hline \multirow[t]{2}{*}{ Bundesministerium der Finanzen (BMF) } & 7.000 & $\begin{array}{l}\text { Ausstattungshilfe für die Zollverwal- } \\
\text { tungen dritter Staaten zur Bekämpfung } \\
\text { der internationalen Rauschgiftkrimina- } \\
\text { lität }\end{array}$ & Bundeshaushalt & 01.2 \\
\hline & 28.700 .000 & $\begin{array}{l}\text { Zollfahndungsdienst: Verhinderung und } \\
\text { Verfolgung des internationalen Drogen- } \\
\text { schmuggels }\end{array}$ & Angabe des Ministeriums & 01.1 \\
\hline $\begin{array}{l}\text { Bundesministerium für } \\
\text { Bildung und Forschung (BMBF) }\end{array}$ & 3.620 .000 & $\begin{array}{l}\text { Suchtforschungsverbünde Bayern/ } \\
\text { Sachsen (ASAT) und } \\
\text { Nordrhein-Westfalen }\end{array}$ & Angabe des Ministeriums & 07.5 \\
\hline $\begin{array}{l}\text { Bundesministerium für Familie, Senioren, } \\
\text { Frauen und Jugend (BMFSFJ) }\end{array}$ & 91.194 & $\begin{array}{l}\text { Personalstelle im Zusammenhang mit } \\
\text { Missbrauch und Abhängigkeit von illega- } \\
\text { len Drogen (Personal- und Sachkosten- } \\
\text { pauschale) }\end{array}$ & Angabe des Ministeriums & 07.6 \\
\hline Bundesministerium der Justiz (BMJ) & 42.033 & $\begin{array}{l}\text { Personalkosten für eine halbe Referen- } \\
\text { tenstelle }\end{array}$ & Angabe des Ministeriums & 03.6 \\
\hline $\begin{array}{l}\text { Bundesministerium für wirtschaftliche } \\
\text { Zusammenarbeit und Entwicklung (BMZ) }\end{array}$ & 11.578 .000 & $\begin{array}{l}\text { Aufwendungen für Drogenbekämpfung } \\
\text { bzw. für Maßnahmen in der Drogen- } \\
\text { politik (Projekte in Entwicklungs- und } \\
\text { Übergangsländern) }\end{array}$ & Angabe des Ministeriums & 01.2 \\
\hline SUMME & 48.055 .227 & & & \\
\hline
\end{tabular}

\section{Gerichte}

Für den Bereich „Gerichte“ erbrachte der drogenbezogene Anteil der Anzahl abgeurteilter Personen, angelegt an die Gesamtausgaben, Ausgaben im Zusammenhang mit illegalen Drogen in Höhe von 1,20 Mrd.€.

\section{Strafvollzug}

Für den Bereich „Strafvollzug“ ergaben sich aus den vorliegenden Daten drei alternative Schätzwerte für die Ausgaben in diesem Bereich:

(1) Der drogenbezogene Anteil der Tage in Haft, angelegt an die staatlichen Gesamtausgaben für „Strafvollzug“ erbrachte Ausgaben in Höhe von 849,5 Mio.€.

(2)Der drogenbezogene Anteil der Anzahl Strafgefangener zum Stichtag 31.3.2006, angelegt an die staatlichen Gesamtausgaben für „Strafvollzug“ ergab einen Betrag von 557,6 Mio.€.

(3)Der drogenbezogene Anteil der Anzahl Strafgefangener am 31.3.2006 multipliziert mit dem durchschnittlichen Tageshaftkostensatz von 91,71€ und hochgerechnet auf 365 Tage erbrachte eine Summe von 486,3 Mio.€.

- Tab. 2 fasst noch einmal alle ermittelten Ausgaben(intervalle) im Verantwortungsbereich der Länder zusammen.

\section{Kommunen}

Die Befragung der kommunalen Spitzen- und Landesverbände erbrachte einen mäßigen Rücklauf (19 von 47=40\%) und die meisten der antwortenden Verbände gaben an, über keine entsprechend detaillierten Daten zu verfügen oder keine Ressourcen für eine Aufstellung der Ausgaben zur Verfügung zu haben. Drei Kommunalverbände konnten genauere Angaben machen, die zeigen, dass ein Großteil der Ausgaben auf kommunaler Ebene im Bereich der Sozialhilfe vermutet werden kann. Die angegebenen Beträge können jedoch nicht als repräsentativ für alle
Tab. 2 Ausgaben der Länder im Zusammengang mit illegalen Drogen für das Jahr 2006.

\begin{tabular}{|c|c|c|}
\hline Ausgabenbereich & $\begin{array}{l}\text { Höhe der } \\
\text { Ausgaben }\end{array}$ & COFOG \\
\hline $\begin{array}{l}\text { "Suchtbudgets“ in den } \\
\text { Landeshaushalten }\end{array}$ & 45,9-73,1 Mio.€ & $\begin{array}{l}07.2,07.4,07.5 \\
07.6,10.4,10.5,10.7\end{array}$ \\
\hline $\begin{array}{l}\text { Maßregelvollzug §64 StGB } \\
\text { (ohne „Trunksucht“) }\end{array}$ & 159,9 Mio.€ & 07.3 \\
\hline Polizei & 1,68-2,17 Mrd. $€$ & 03.1 \\
\hline Gerichte & 1,20 Mrd.€ & 03.3 \\
\hline Strafvollzug & $\begin{array}{l}\text { 486,3- } \\
849,5 \text { Mio.€ }\end{array}$ & 03.4 \\
\hline SUMME & $\begin{array}{l}3,57-4,45 \\
\text { Mrd.€ }\end{array}$ & \\
\hline
\end{tabular}

Kommunalverbände angesehen werden. Somit fehlen auf kommunaler Ebene belastbare Daten zu Ausgaben im Zusammenhang mit illegalen Drogen.

Für den Teilbereich der kommunalen Ausgaben für die ambulante Suchthilfe konnte anhand der Daten aus der Deutschen Suchthilfestatistik [12] eine Schätzung vorgenommen werden: Kommunale Mittel machen in den teilnehmenden Einrichtungen $52,8 \%$ des Budgets aus. Das durchschnittliche Budget einer Einrichtung betrug im Jahr $2006312.446 €$. Hochgerechnet auf alle 934 ambulanten Suchthilfeeinrichtungen, die es laut einer Schätzung von 2005 in Deutschland gibt [14] ergeben sich somit gerundet 154,1 Mio€. Der Anteil illegaler Drogen an dieser Summe kann anhand der angegebenen Hauptdiagnosen der behandelten Patienten mit 33,6\% angesetzt werden. Somit resultieren kommunale Ausgaben für die ambulante Suchthilfe (COFOG 07.2) in Höhe von etwa 51,8 Mio.€. 


\section{Sozialversicherungsträger}

Im Folgenden werden die Ergebnisse zu den Ausgaben der Sozialversicherungsträger im Zusammenhang mit illegalen Drogen aufgezeigt. Eine wesentliche Rolle bei der Identifikation der Ausgaben bei den Sozialversicherungsträgern spielt die Klassifikation der Krankheitsdiagnosen nach dem international verwendeten, auf Deutschland angepassten International Classification of Diseases (ICD) -10-Code. Die relevanten ICD-10-Codes im Zusammenhang mit illegalen Drogen sind F11.x (Psychische und Verhaltensstörungen durch Opioide), F12.x (Psychische und Verhaltensstörungen durch Cannabinoide), F13.x (Psychische und Verhaltensstörungen durch Sedativa oder Hypnotika), F14.x (Psychische und Verhaltensstörungen durch Kokain), F15.x (Psychische und Verhaltensstörungen durch andere Stimulanzien, einschließlich Koffein), F16.x (Psychische und Verhaltensstörungen durch Halluzinogene), F18.x (Psychische und Verhaltensstörungen durch flüchtige Lösungsmittel), F19.x (Psychische und Verhaltensstörungen durch multiplen Substanzgebrauch und Konsum anderer psychotroper Substanzen). Diese Klassifikation orientiert sich an der Auswahl der EBDD [15].

Rentenversicherungen

Insgesamt lassen sich die Ausgaben der Rentenversicherungen im Zusammenhang mit illegalen Drogen auf etwa 171,7 Mio.€ beziffern ( 0 Tab. 3$)$.

Tab. 3 Ausgaben der Rentenversicherungen im Zusammenhang mit illegalen Drogen für das Jahr 2006.

\begin{tabular}{|c|c|c|c|}
\hline Ausgabenart & Anzahl Fälle & Höhe der Ausgaben & COFOG \\
\hline $\begin{array}{l}\text { medizinische } \\
\text { Rehabilitation } \\
\text { (ambulant) }\end{array}$ & 11.678 & $112.275 .400 €$ & 07.2 \\
\hline $\begin{array}{l}\text { medizinische Reha- } \\
\text { bilitation (stationär) }\end{array}$ & 350 & $366.670 €$ & 07.3 \\
\hline $\begin{array}{l}\text { Leistungen zur } \\
\text { Teilhabe am Arbeits- } \\
\text { leben }\end{array}$ & 201 & $4.013 .040 €$ & 10.5 \\
\hline $\begin{array}{l}\text { Renten wegen } \\
\text { Erwerbsminderung }\end{array}$ & 6.219 & $55.088 .650 €$ & 10.1 \\
\hline Modellprojekte & 0 & - & 07.5 \\
\hline SUMME & & 171.743.760€ & \\
\hline
\end{tabular}

Die Rentenversicherung Bund berichtete zusammenfassend für alle Rentenversicherungen in Deutschland 11.678 Fälle für die ambulante medizinische Rehabilitation mit Ausgaben in Höhe von 112.275.400€ sowie bei 350 angegebenen Fällen für die stationäre medizinische Rehabilitation Kosten in Höhe von 366.670€. Die durchschnittliche Dauer der Inanspruchnahme über alle Diagnosen beträgt für die ambulante Rehabilitation 96 Tage und für die stationäre Rehabilitation 106 Tage. Für Leistungen zur Teilhabe am Arbeitsleben ergaben sich Ausgaben in Höhe von 4.013.040€, die durch 201 Personen beansprucht wurden. 21 verschiedene Maßnahmearten aus dem Maßnahmenkatalog für Leistungen zur Teilhabe am Arbeitsleben wurden von dieser Klientel in Anspruch genommen. Die Renten aufgrund von Erwerbsminderung wurden in einer Höhe von 55.088.650€ errechnet. 6.219 Rentner wiesen eine relevante FDiagnose als Erstberentungsdiagnose auf. Das durchschnittliche Alter bei Rentenzugang lag hier bei 45 Jahren, das sind 5 Jahre unter dem Durchschnittsalter bei der Betrachtung aller Diagnosen. Im Jahr 2006 ist die Anzahl der Fälle mit den relevanten Erstberentungsdiagnosen bei Männern (414) dreifach so hoch wie bei den Frauen (145).

Es wurden 2006 zwei Modellprojekte für Drogenabhängige unterstützt. Es fielen jedoch keine Kosten diesbezüglich bei den Rentenversicherungen an.

Gesetzliche Krankenversicherung

Insgesamt lässt sich die Schätzung der Ausgaben der gesetzlichen Krankenkassen im Zusammenhang mit illegalen Drogen auf ca. 1,4 Mrd. € beziffern ( $\bullet$ Tab. 4).

Die Ausgaben für Krankenhausaufenthalte in Allgemeinen Krankenhäusern (ohne Aufenthalte in psychiatrischen Abteilungen) mit den relevanten F-Diagnosen als Hauptdiagnosen lassen sich auf ca. 6,4 Mio.€ für das Jahr 2006 schätzen. In diese Analyse miteinbezogen wurden die Fälle mit folgenden Diagnosis Related Groups (DRGs): V61Z - Drogenintoxikation und -entzug (1.541 Fälle), V63Z - Störungen durch Opioidgebrauch und Opioidabhängigkeit (1.899 Fälle), V64Z - Störungen durch anderen Drogengebrauch und Medikamentenmissbrauch und andere Drogen- und Medikamentenabhängigkeit (2.645 Fälle) und V65Z - Humanes Immundefizienz-Virus (HIV)-Krankheit bei Alkohol- und Drogengebrauch und Alkohol- und drogenindu-

Tab. 4 Ausgaben der GKV im Zusammenhang mit illegalen Drogen für das Jahr 2006.

\begin{tabular}{|c|c|c|c|c|}
\hline Ausgabenart & & Rücklaufquote $^{2}$ & Ausgaben & COFOG \\
\hline Krankenhausaufenthalte & & - & $6.406 .000 €$ & 07.3 \\
\hline \multirow[t]{2}{*}{ Psychiatrie } & & $57 \%$ & $232.760 .000 €$ & 07.3 \\
\hline & Methadon + Levomethadon & $44 \%$ & $32.183 .000 €$ & \\
\hline Medikation $^{3}$ & Naltrexon & $51 \%$ & $453.000 €$ & 07.1 \\
\hline ambulante Arztkontakte & Buprenorphin & $57 \%$ & $22.537 .000 €$ & \\
\hline Kontakte zu Substitutionsärzten & & $32 \%$ & $167.002 .000 €$ & 07.2 \\
\hline medizinische Rehabilitation & & $38 \%$ & $742.000 .000 €$ & 07.2 \\
\hline Notarzteinsätze & & $46 \%$ & $27.740 .000 €$ & 07.3 \\
\hline Modellprojekte & & $26 \%$ & $4.751 .000 €$ & 07.3 \\
\hline Prävention und Selbsthilfe & & $4 \%$ & $-€$ & 07.5 \\
\hline Krankengeld & & $19 \%$ & $536.000 €$ & 07.4 \\
\hline Soziotherapie & & $51 \%$ & $25.701 .000 €$ & 10.1 \\
\hline Heilmittel & & $14 \%$ & $580.000 €$ & 07.6 \\
\hline \multirow[t]{2}{*}{ Hilfsmittel } & & $6 \%$ & $33.543 .000 €$ & 07.1 \\
\hline & & $2 \%$ & $106.000 .000 €$ & 07.1 \\
\hline GESAMT & & & $1.402 .000 .000 €$ & \\
\hline
\end{tabular}


zierten psychischen Störungen (45 Fälle). Die Ausgaben für Aufenthalte in Psychiatrischen Krankenhäusern bzw. Abteilungen im Zusammenhang mit illegalen Drogen belaufen sich auf ca. 232,8 Mio.€ im Jahr 2006.

25 der 40 angeschriebenen gesetzlichen Krankenkassen haben einen teilweise oder vollständig beantworteten Fragebogen zurückgesandt. Es lagen Angaben bezüglich der Ausgaben zu 40.714.953 Versicherten vor, die 58\% der Gesamtversicherten der gesetzlichen Krankenversicherung darstellen. Die relevanten F-Diagnosen stellten hier die Aufnahme- bzw. Hauptdiagnose dar. Als spezifische Medikationen wurden die Ausgaben für Methadon, Levomethadon, Naltrexon und Buprenorphin in die Analyse miteinbezogen. Die Verschreibungen für Methadon und Levomethadon beanspruchen ca. 32,2 Mio.€, für Naltrexon ca. $453.000 €$ und für Buprenorphin ca. 22,5 Mio€. Ambulante Arztkontakte aufgrund illegaler Drogen kosteten schätzungsweise ca. 167 Mio. €. Durch Kontakte zu Substitutionsärzten fielen Kosten in Höhe von ca. 742 Mio.€ für die gesetzliche Krankenversicherung an. Die medizinische Rehabilitation von Patienten mit illegalem Drogenkonsum beansprucht ca. 27,7 Mio.€. Auch hier entsprachen die Aufnahme- bzw. Hauptdiagnosen den relevanten F-Diagnosen.

Die Notarzteinsätze im Zusammenhang mit illegalen Drogen kosteten die gesetzlichen Krankenversicherungen im Jahr 2006 ca. 4,8 Mio€. Keine der teilnehmenden Krankenkassen teilte die Durchführung eines Modellprojektes im Zusammenhang mit illegalen Drogen im Jahr 2006 mit. Für Prävention und Selbsthilfe bezogen auf illegale Drogen konnten Ausgaben in Höhe von ca. $536.000 €$ errechnet werden. Krankengeld wegen illegaler Drogen wurde von den gesetzlichen Krankenkassen ca. 25,7 Mio.€ gezahlt. Soziotherapie-Leistungen im Zusammenhang mit illegalen Drogen beanspruchten ca. 580.000€ im Jahr 2006. Die ermittelten Ausgaben für Heil- und Hilfsmittel beliefen sich auf ca. 33,5 Mio.€ bzw. ca. 106 Mio.€ im Jahr 2006.

Die Gesamtausgaben der Sozialversicherungen im Zusammenhang mit illegalen Drogen betragen somit ca. 1.574.000.000€ für das Jahr 2006.

\section{Zusammenfassung der Ergebnisse}

Zusammengefasst ergibt sich aus den verschiedenen Bereichen eine Gesamtsumme der öffentlichen Ausgaben für illegale Drogen in Höhe von 5,2 bis 6,1 Mrd. €. Bei diesem Betrag handelt es sich tendenziell um eine Unterschätzung der wahren Ausgaben, da in einigen Bereichen keine Informationen vorlagen und für die Berechnungen in anderen Bereichen eher konservative Schätzwerte herangezogen wurden.

Für eine inhaltliche Betrachtung wurden die ermittelten Ausgaben den Kategorien der COFOG-Klassifikation zugeordnet: Die drogenbezogenen Ausgaben für öffentliche Sicherheit und Ordnung (COFOG-Funktion 03) liegen bei 3,4-4,4 Mrd.€ und machen mit 65-70\% den größten Teil der ermittelten Staatsausgaben in Bezug zu illegalen Drogen aus. Die Ausgaben für Gesundheitswesen und soziale Sicherung (COFOG-Funktionen 07 und 10) wurden zusammengefasst, da v.a. in den Länderhaushalten häufig keine eindeutige Zuordnung möglich war. Sie haben mit einer Höhe von 1,8 bis 1,9 Mrd.€ einen Anteil von 30-35\% an den Gesamtausgaben in Zusammenhang mit illegalen Drogen. Der Anteil der Ausgaben für allgemeine öffentliche Verwaltung (COFOG-Funktion 01; hier vor allem die Ausgaben für Entwicklungshilfe und Zollfahndung) liegt mit 40,2 Mio.€ bei unter $1 \%$. Alle ermittelten Ausgaben konnten einer dieser vier Funktionen zugeordnet werden, Ausgaben in Bezug zu illegalen Drogen in anderen Funktionsbereichen (z.B. COFOG-Funktion 09 „Bildungswesen“) konnten nicht identifiziert werden oder waren nur so vereinzelt dargestellt, dass sie hier außer Acht gelassen wurden. Es liegt jedoch die Vermutung nahe, dass auch in anderen Bereichen weitere Ausgaben verborgen sind.

Vergleicht man die Ausgaben, die speziell für den Bereich illegale Drogen getätigt werden, mit den Gesamtausgaben für die jeweilige Staatsfunktion, ergibt sich folgendes Bild: Die Ausgaben mit Bezug zu illegalen Drogen machen im Bereich „allgemeine öffentliche Verwaltung“ (Gesamtausgaben: 129,6 Mrd.€) $0,03 \%$ der Ausgaben aus. Im Bereich öffentliche Sicherheit und Ordnung (Gesamtausgaben: 37,1 Mrd.€) stehen etwa 9,1-11,4\% der Ausgaben im Bezug zu illegalen Drogen, im Gesundheitsund Sozialwesen (Gesamtausgaben: 642,2 Mrd.€) dagegen nur ungefähr $0,3 \%$.

\section{Diskussion \\ $\nabla$}

Erste Ergebnisse zur Schätzung der Ausgaben aufgrund des Konsums illegaler Drogen wurden in einem interdisziplinären Experten-Workshop diskutiert. Unterschiedliche methodische Anregungen zur Vorgehensweise wurden bei der Berechnung der hier vorgestellten Ergebnisse bereits umgesetzt. Einige Besonderheiten werden im Folgenden diskutiert:

Ein allgemeines Problem bei der Datenerhebung, vor allem auf Ebene der Gebietskörperschaften, bestand darin, dass bei den gekennzeichneten Ausgaben häufig keine Differenzierung zwischen legalen und illegalen Drogen vorgenommen wurde. Dies basiert auf der Entscheidung, Sucht in Deutschland auf politischer Ebene als Gesamtphänomen und nicht getrennt nach Substanzen zu betrachten. Somit musste der jeweilige Anteil illegaler Drogen an den Ausgaben häufig geschätzt werden. Da wir uns dabei auf Angaben der zuständigen Ministerien verlassen mussten, sind die ermittelten Beträge nicht immer objektiv nachvollziehbar. Auf Ebene der Sozialversicherungsträger spielte dieses Problem aufgrund der Verwendung von ICD-10-Codes keine so große Rolle, wobei auch hier die Schwierigkeit besteht, dass die Aufteilung anhand von Stoffgruppen und nicht anhand von Legalitätskriterien geschieht. Somit wurden auch Diagnosen in die Datenerhebung eingeschlossen, die zum Teil die Abhängigkeit von Medikamenten beinhalten, die nicht zu den illegalen Drogen zählen. Dieses Vorgehen kann jedoch dadurch gerechtfertigt werden, dass es zum einen im Sinne der Vergleichbarkeit dem vorgeschlagenen Prozedere der EBDD entspricht [15] und zum anderen auf diese Weise keine wesentlichen Gruppen von Drogen systematisch ausgeschlossen wurden. Sicherlich wäre es in Zukunft interessant, bei entsprechender Verfügbarkeit von differenzierten Daten, genauere Berechnungen vorzunehmen. Weitere methodische Schwierigkeiten erwuchsen aus der haushaltswirtschaftlichen Unabhängigkeit der Länder. Es bestanden große Unterschiede zwischen den Haushaltsdokumenten der Länder in Bezug auf die Systematik, den Grad der Detailliertheit und die Verwendung von Begrifflichkeiten. Somit gestaltete sich das Durchsuchen der Haushaltspläne sehr aufwendig. Die gefundenen Daten sind sehr heterogen, wobei nicht immer klar zu erkennen ist, zu welchem Teil dies an der Beschaffenheit der Haushaltsdokumentation und zu welchem Teil an tatsächlichen Unterschieden zwischen den Ländern liegt. Auch das Subsidiaritätsprinzip wird von den Ländern unterschiedlich stark genutzt und eine Weitergabe von Mitteln in die Verantwortung der Kommunen wird in den Haushalten nicht immer deutlich ge- 
kennzeichnet. Somit besteht prinzipiell die Gefahr einer Doppelerfassung von Ausgaben. In der vorliegenden Studie ist das damit verbundene Risiko einer Überschätzung von Kosten dadurch minimiert, dass auf kommunaler Ebene kaum verwendbare Daten vorlagen. Dies stellt jedoch gleichzeitig eine wesentliche Einschränkung der Arbeit dar: Da für den kommunalen Bereich kaum belastbare Zahlen zu identifizieren waren, muss hier von einer Unterschätzung der Ausgaben ausgegangen werden. Die Größenordnung lässt sich momentan jedoch nicht abschätzen.

Das Vorgehen bei der Schätzung der Ausgaben für Polizei, Gerichte und Strafvollzug unterschied sich wesentlich vom Vorgehen bei anderen Ausgabenbereichen, indem zum großen Teil Sekundärdaten dafür herangezogen wurden. Diese Methodik beruht auf vielen Annahmen und bringt so ganz eigene Probleme mit sich [5], hat aber auch Vorteile: Erstens lagen für diese Funktionen kaum Daten in den Haushaltsplänen vor, die die Zuordnung zum Drogensektor ermöglicht hätten. Ohne das Zurückgreifen auf andere verfügbare Daten hätte somit ein wesentlicher Teil öffentlicher Ausgaben in Zusammenhang mit illegalen Drogen keinen Eingang in die hier berichtete Summe gefunden. Zweitens orientierte sich das Vorgehen an einer der wenigen vorhandenen Studien [5] und ermöglicht so einen Vergleich zu früher ermittelten Ausgaben. Drittens ist das Vorgehen transparent und basiert im Gegensatz zu den Schätzwerten, die auf Angaben von Ministerien beruhen, auf öffentlich zugänglichen Daten und kann objektiv nachvollzogen werden.

Bei der Schätzung der Ausgaben der Rentenversicherung kann man insgesamt von einer verhältnismäßig hohen Validität ausgehen. Es konnten alle relevanten Ausgabenarten mit in die Schätzung einbezogen werden, da die Rentenversicherung Bund für alle Ausgabenarten Daten für die Schätzung zur Verfügung gestellt hat. Die Höhe der Ausgaben ist mit ein paar Abstrichen aufgrund der Verwendung von Mittelwerten als realistisch einzuschätzen.

Eine generelle Problematik beim Abruf von Routinedaten der Krankenkassen stellt die unterschiedliche Datenstruktur und differenzierung der einzelnen Kassen dar. Für die Beantwortung der einzelnen Fragen des Fragebogens benötigte jede Kasse andere Indikatoren.

Bei den Schätzungen der Ausgaben der gesetzlichen Krankenversicherung handelt es sich um Hochrechnungen. Hierbei wurde eine als repräsentativ zu bezeichnende Stichprobe befragt. Insgesamt ist eine durchschnittliche Rücklaufquote von $63 \%$ der befragten Krankenkassen zufriedenstellend. Damit enthält der vorliegende Datensatz Angaben zu 58\% aller GKV-Versicherten. Doppelerfassungen von Ausgaben hätten ggf. im Rahmen von Leistungen gemäß $§ 264$ SGB V auftreten können. Leistungen von Nicht-Versicherten, die Sozialhilfe bekommen, wurden in den Krankenkassen-Daten mit erfasst, obwohl die Kassen Leistungen nach $\S 264$ von den Sozialhilfeträgern zurückerstattet bekommen (d.h. entsprechende Zahlen müssten eigentlich unter den Ausgaben für Sozialhilfe erfasst werden). Aufgrund der Nichterfassung der kommunalen Ausgaben für Sozialhilfe kommt es hier jedoch zu keiner Überschätzung.

Insgesamt muss bei der hier vorliegenden Schätzung der Ausgaben der gesetzlichen Krankenkassen im Zusammenhang mit illegalen Drogen eher von einer Unterschätzung der Ausgaben ausgegangen werden, da differenziertere Abfragen wie die Erfassung bspw. von Nebendiagnosen im Zusammenhang mit dem Konsum von illegalen Drogen oder der Notarzteinsatz ohne nachfolgenden Krankenhausaufenthalt aufgrund des damit verbundenen Aufwandes für die befragten Krankenkassen bzw. der fehlenden Möglichkeit der Zuordnung zum Konsum von illegalen Drogen nicht erfasst wurden.

Bei der inhaltlichen Betrachtung der ermittelten Ausgaben zeigt sich, dass ein Großteil des (finanziellen) Engagements des Staates in repressive Maßnahmen zur Bekämpfung von Kriminalität im Zusammenhang mit illegalen Drogen fließt. Bei der Bewertung der Höhe dieses Anteils (bei der hier ermittelten Summe ca. $65-70 \%)$ muss jedoch beachtet werden, dass insbesondere für den Bereich der sozialen Sicherung die tatsächlichen Ausgaben vermutlich um einiges höher liegen als die hier berichteten Beträge. Auch für die Funktion Bildungswesen fehlen Informationen. Somit würden sich die Anteile an der Gesamtsumme bei einer besseren Datenlage noch verschieben. Es erscheint jedoch beachtlich, dass etwa 10\% der gesamten öffentlichen Ausgaben für die öffentliche Sicherheit und Ordnung einen Bezug zu illegalen Drogen aufweisen, während in anderen Funktionen (wie Gesundheits- und Sozialwesen) die Beschäftigung mit illegalen Drogen nur einen sehr kleinen Anteil der Gesamtausgaben bedingt.

Das Ziel des Projektes, eine erste Aufstellung wesentlicher Ausgaben für den Missbrauch und die Abhängigkeit von illegalen Drogen vorzunehmen, konnte erreicht werden. Anzumerken ist, dass zweifelsohne Unsicherheiten bezüglich des Gesamtergebnisses aufgrund der heterogenen Datenqualität bestehen. Die Methodenentwicklung steht hier nicht nur in Deutschland, sondern in ganz Europa noch am Anfang.

Das Ergebnis gibt noch keinen Aufschluss über die Angemessenheit und den tatsächlichen Nutzen der aufgewendeten öffentlichen Mittel. Es bildet allerdings die unabdingbare Grundlage für eine solche Bewertung und leistet einen wichtigen Beitrag zur Versachlichung der Diskussion und zu einer rationalen Politikentwicklung.

\section{Förderhinweis}

$\nabla$

Diese Publikation ist im Rahmen des Projektes „Illegale Drogen - Eine Schätzung der direkten Ausgaben der öffentlichen Haushalte in der Bundesrepublik Deutschland“ entstanden, das vom Bundesministerium für Gesundheit (Kennzeichen: 2508DSM401) finanziell gefördert wurde.

\section{Literatur}

1 Bundesministerium für Gesundheit, Drogen- und Suchtbericht Mai 2008. Online verfügbar unter: http://www.bmg.bund.de/cln_110/SharedDocs/Downloads/DE/Drogen-Sucht/Drogen_20und_20Sucht_20allgemein/Drogen-_20und_20Suchtbericht_202009,templateId=raw,prope rty=publicationFile.pdf/Drogen-\%20und\%20Suchtbericht\%202009.pdf (30.03.2009)

2 Official Journal of the European Union (OJ). C168 of 08.07.2005: 17

3 European Monitoring Center for Drugs and Drug Addiction. EMCDDA 2008 Selected Issue - Towards a better understanding of drug-related public expenditure in Europe. Luxembourg: Office for Official Publications of the European Communities; 2008; 1-28

4 Simon $R$ et al. Bericht des Nationalen REITOX Knotenpunkts für Deutschland an die EBDD: Drogensituation 2001; 2002: 122-132

5 Hartwig KH, Pies I. Rationale Drogenpolitik in der Demokratie: Wirtschaftswissenschaftliche und wirtschaftsethische Perspektiven einer Heroinvergabe. Tübingen: J. C. B. Mohr; 1995; 20-34

6 Schöffski O, von der Schulenburg JM. Gesundheitsökonomische Evaluationen 3. Aufl. Springer Verlag: Heidelberg; 2008; 4

7 Eurostat. Ausgaben des Staates nach Ausgabenbereich (COFOG), 2008 Online verfügbar unter: http://epp.eurostat.ec.europa.eu/portal/ page?_pageid=1073,46870091\&_dad=portal\&_schema=PORTAL\&p_ product_code=GOV_A_EXP (30.03.2009) 
8 Bundeskriminalamt, Hrsg. Polizeiliche Kriminalstatistik Bundesrepublik Deutschland. Berichtsjahr 2006. Wiesbaden: Bundeskriminalamt; 2007; 1-471

9 Statistisches Bundesamt. Rechtspflege. Strafverfolgung. Fachserie 10, Reihe. Wiesbaden: Statistisches Bundesamt; 2007; 1-484

10 Statistisches Bundesamt. Rechtspflege. Strafvollzug - Demografische und kriminologische Merkmale der Strafgefangenen zum Stichtag 31.3. Fachserie 10, Reihe 4.1. Wiesbaden: Statistisches Bundesamt; 2006; $1-32$

11 Graf von der Schulenburg JM, Claes C. Das bundesdeutsche Modellprojekt zur heroingestützten Behandlung Opiatabhängiger - Eine multizentrische, randomisierte, kontrollierte Therapiestudie. Gesundheitsökonomische Begleitforschung. Endbericht; 2006; 1-76

12 Sonntag D, Bauer C, Hellwich AK. Suchthilfestatistik 2006 für Deutschland. Tabellenband für ambulante Einrichtungen. München: IFT Institut für Therapieforschung; 2007, Online verfügbar unter: http:// www.suchthilfestatistik.de/ambulant.htm (30.03.2009)
13 European Commission. Manual on sources and methods for the compilation of COFOG Statistics - Classification of the Functions of Government (COFOG). ications of the European Communities 2007, Online verfügbar unter: http://epp.eurostat.ec.europa.eu/cache/ITY_ OFFPUB/KS-RA-07-022/EN/KS-RA-07-022-EN.PDF (30.03.2009)

14 Simon R. Analyse der Länderkurzberichte zum Umfang substanzbezogener Störungen und zur Betreuungssituation 2004. München: DBDD; 2005

15 European Monitoring Centre for Drugs and Drug Addiction. Standard Table 1. Standardized results and methodology of adults national population surveys on drug use 2008, Online verfügbar unter: http:// www.emcdda.europa.eu/attachements.cfm/att_67103_EN_EMCDDAGPS-ST1.pdf (30.03.2009) 